\title{
Puppet Attack: A Denial of Service Attack in Advanced Metering Infrastructure Network
}

\author{
Ping $\mathrm{Yi}^{*}$, Ting Zhu ${ }^{\dagger}$, Qingquan Zhang ${ }^{\dagger}$, Yue $\mathrm{Wu}^{*} \mathrm{Li} \mathrm{Pan}^{*}$ \\ *National Engineering Laboratory for Information Content Analysis Technology, \\ School of Information Security Engineering, Shanghai Jiao Tong University, Shanghai, 200240, China \\ ${ }^{\dagger}$ Department of Computer Science and Electrical Engineering, University of Maryland, Baltimore County, MD 21250, USA
}

\begin{abstract}
Advanced Metering Infrastructure (AMI) is the core component in a smart grid that exhibits a highly complex network configuration. AMI shares information about consumption, outages, and electricity rates reliably and efficiently by bidirectional communication between smart meters and utilities. However, the numerous smart meters being connected through mesh networks open new opportunities for attackers to interfere with communications and compromise utilities assets or steal customers private information.

In this paper, we present a new DoS attack, called puppet attack, which can result in denial of service in AMI network. The intruder can select any normal node as a puppet node and send attack packets to this puppet node. When the puppet node receives these attack packets, this node will be controlled by the attacker and flood more packets so as to exhaust the network communication bandwidth and node energy. Simulation results show that puppet attack is a serious and packet deliver rate goes down to $20 \%-10 \%$. After analyzing the puppet attack, we propose the detection and prevention mechanism. Simulations show that puppet attack causes the same damage as a flooding attack and the proposed method can prevent the puppet attack efficiently.
\end{abstract}

\section{INTRODUCTION}

Smart grid delivers electricity between suppliers and consumers using two-way digital technology to control intelligent appliances at consumers home or building to save energy, reduce cost and increase reliability, efficiency and transparency [1]. Advanced Metering Infrastructure (AMI) is one of the major advancement for collecting data on energy consumption more frequently and accurately. The role of an AMI is to enable communication between utility companies and electricity meters, including remote electricity usage readings (on-demand and periodic), sending of updated price information to the meters, transmission of alerts about outages, and upgrades of meter firmware, among other communications. Some messages require real-time delivery, while others can be buffered and delayed without negative consequences [2].

Advanced Metering Infrastructure(AMI) refers to systems that collect, measure and analyze energy usage, from networks that connected to next-generation electricity meters, or, smart meters. AMI includes software, hardware, communication networks, customer-associated systems and meter data management software. Wireless mesh network is one key technology in AMI network [3]. The smart meters form a mesh network and multi-hop communication is possible through several meters until the concentrator is reached. Wireless mesh network is able to handle smart metering communication traffic with a high reliability, and the potential coverage gaps are properly filled with repeater nodes [4], [5].

The addition of a communication infrastructure and the new computational capabilities of smart grid devices add a significant attack surface to traditional energy delivery systems. For example, cyber intrusions that would previously have required physical access to the utility network may now be possible through a remote exploit. In the context of AMIs, the fact that smart meters are not only connected to the utility network but also directly accessible by customers enables new attack vectors. Indeed, field area networks in which meters are deployed appear to be an attractive target for adversaries, because they consist of large numbers of physically accessible devices and have limited or no security monitoring capabilities. Security threats grow exponentially, from inside and outside of the AMI network system [6].

In this paper, we present a new attack called a puppet attack, which results in denial of service in AMI network. In this attack, the attacker first selects one or more normal nodes as puppets. Then the attacker sends out data packets, which contain specific attack information to these puppet nodes. When the puppet nodes receive these attack packets, they generate a high volume of route packets. There is limited communication bandwidth in mesh network and route packets are top priority in all packets. Excess route packets will exhaust limited communication bandwidth and result in network congest. As a result, puppet attack causes a denial of service attack in AMI network.

The contributions of this paper are as follows.

- We identify and present a new and severe denial of service attack (i.e., puppet attack) in AMI network.

- This work is the first in-depth work to investigate the impact of a puppet attack in AMI. We analyze the mechanism of puppet attack and compare it with the other attack.

- We present a distributed and adaptive method to detect the puppet attack. Then an isolation mechanism is designed to prevent the puppet attack. In this mechanism, the direct neighbors of the attacker cut off the links to the attacker and do not receive the packets from the attacker any more. 
The remainder of this paper is structured as follows. Section II introduces the puppet attack in AMI network. Section III describes the approach to detect and prevent the puppet attack. In Section IV, we present simulation experiments. Section V briefly discusses related work, and Section VI concludes the paper.

\section{THE MOdEL OF THE PUPPET ATTACK}

\section{A. Overview of Advanced Metering Infrastructure (AMI)}

In smart grid, electricity suppliers can monitor, predicate, and control energy generation consumption in real time. Users can know the current price of electrical energy and obtain energy management information from smart meters. It helps users reduce homes energy use. AMI network is the core component to help energy information to transfer and access.

Advanced metering infrastructure (AMI) is an architecture for automated, two-way communication between a smart utility meter with communication network and a utility company. The goal of an AMI is to provide utility companies with realtime data about power consumption and allow customers to make informed choices about energy usage based on the price at the time of use. AMI is an important part of any smart grid initiative.

Wireless mesh network technology is uniquely suited for use in AMI applications due to its ability to dynamically form ad hoc network links between neighboring network nodes. Furthermore communication range can be increased by performing multiple hops from one node to the next until the final destination is reached. Thus wireless mesh networks are able to overcome variable propagation conditions by finding alternative paths through the mesh in the event that one path is blocked by an obstruction, e.g. a lorry parked outside a house.

Dynamic Source Routing (DSR) is one of classic route protocols in wireless mesh network. It is used to form mesh network and forward packets. We introduce Dynamic Source Routing protocol and a denial of service attack against the source routing protocol in following section.

\section{B. Dynamic Source Routing (DSR) Protocol}

DSR is an on-demand routing protocol using in wireless mesh network, composed of two parts: route discovery and route maintenance. In DSR, whenever a node need to send a packet to a destination and cannot find a route to that destination in its route cache, the node initiates route discovery. The initiator broadcasts a route request packet(RREQ) to its neighbors, specifying the destination and a unique sequence number from the initiator. A node receives the RREQ, if it has recently received the same request identifier from the initiator, it discards the RREQ. Otherwise, it adds its own node address to the list in the RREQ and rebroadcasts the packet. When the RREQ reaches the destination, the destination sends a route reply packet(RREP), including a list of intermediate node's addresses based on the RREQ. When the RREP reaches the initiator of the RREQ, the initiator caches the new route in its route cache. Intermediate nodes also can send RREPs, if they have routes to the destination.

Route maintenance starts when a node sending a packet along a specified route to the destination discovers that the route is broken. If a node on the route cannot receive the confirmation from its next-hop node after a limited number of retransmissions of the packet, it returns a route error packet(RRER) to the source of the packet, identifying the path from itself to the next-hop node as broken. The source then removes this broken link from its route cache. For successive packets to the destination, the source may select an other route to that destination from its cache, or it may initiate a new route discovery for that destination if no route exists.

Route salvage is a function of Route Maintenance. Suppose that an intermediate node forwards a packet, and detects that the route to the next-hop node for that packet is broken. The node will start to salvage the packet. To salvage a packet, the node first searches for a replacement route to the destination in his cache. If the node finds a route, it replaces the initial route on the packet with the path from its route cache. The node then sends the packet to the next-hop node along this route. If there exists no route in its cache, the node broadcasts an RREQ to find a new route for the destination. When the node receives RREPs, it replaces the initial route for the packet with the new route. In a word, if a node can forward the packets to its next-hop node, it can salvage the packet by replacing the initial route in the packet with this new route, rather than discarding the packet. This salvage mechanism is not secure and is vulnerable to a puppet attack.

\section{Puppet Attack}

In this section, we describe our newly discovered attack, called puppet attack, which is a denial of service attack in AMI network. We first describe the effect of puppet attack. We begin with the normal process of a route discovery in Fig. 1 and then discuss the puppet attack using an example in Fig. 2 .

Discovery process includes two parts: route request and route reply. It is illustrated by Fig.1. For example, there are five nodes: $A, B, C, D, E . A$ is the source node and $E$ is the destination. Fig.1 illustrates the normal process when nodes receive and forward route packets. The right line shows the RREQ process, and the letters indicate the address list in RREQ. When a node receives a RREQ, it adds its own node address to the route in the RREQ and rebroadcasts the RREQ. The right line shows the RREP process, and the letters indicate the address list in RREP.

Fig.2 illustrates an example of a puppet attack. The right line in Fig. 2 is the normal packet forward process from node $A$ to $E$. Assume that node $A$ is the malicious node. When $A$ receives RREP, it produces a specific error route, where the address of node $E$ is deleted from the address list. As a result, A will set up the error path " $A B C D X$ " when it sends the packet. When $A$ sends packets on the error path, the packet reaches node $D$. Node $D$ cannot forward the packet to nexthop node $X$ because node $X$ is not its neighbor. Node $D$ will 


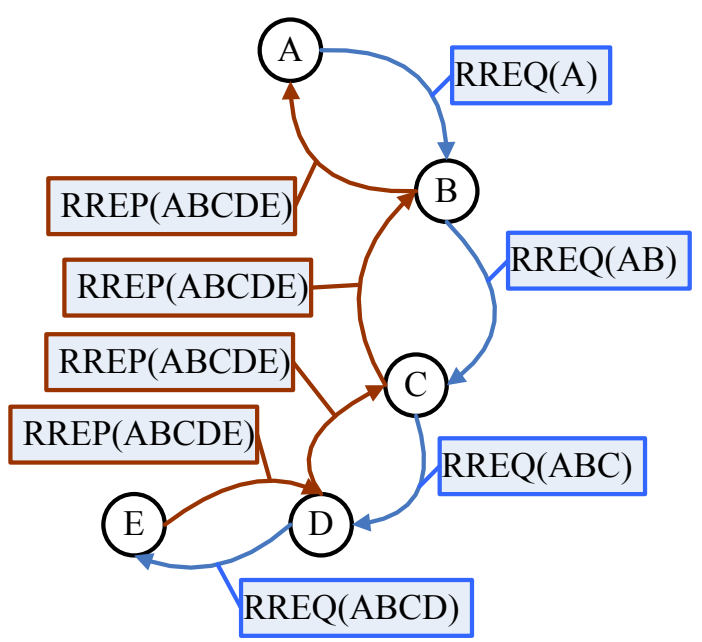

Fig. 1. The address list of packet when forwarding RREQ and replying RREP

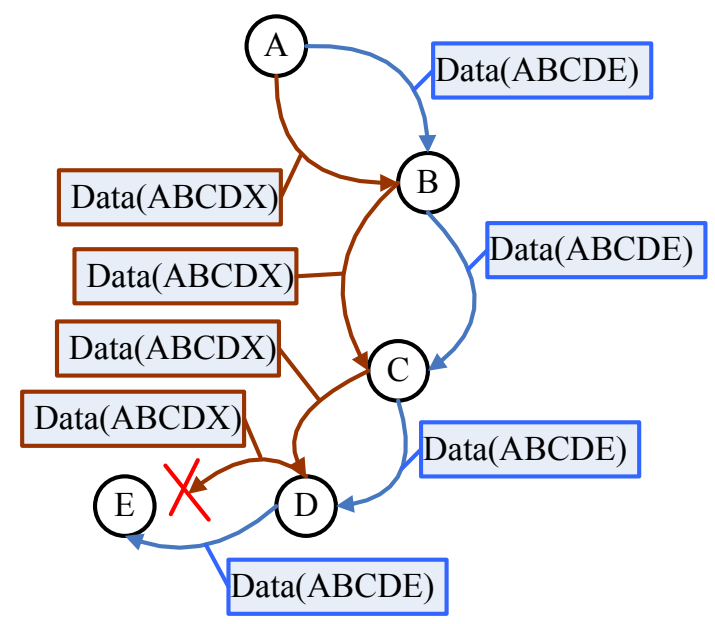

Fig. 2. Malicious node alters route of packet. The right line is a normal route in a packet and the process of forward the packet. The left line is that node A changes route in packet and the packet can cannot be forwarded by node $D$.

begin to salvage the packet instead of discarding it. First, $D$ will search for a replacement route to node $X$ in its cache. If $D$ cannot find a route in its cache, $D$ launches the Route Discovery process to find a new route to $X$. $D$ broadcasts a RREQ to its neighbors and all of its neighbors will forward the RREQ to their neighbor nodes. This results in the flooding of RREQs. If $A$ maliciously changes route $A B C D E$ into $A B C X, A B X$ and send these packets, nodes $B$ and $C$ will start to salvage the packets, which have specific error routes. They broadcast RREQs to find replacement routes. Excessive RREQs exhaust nodes' resource and communication bandwidth. This results in a denial of service.

Algorithm 1 shows the process how to generate an attack packet. 1) Suppose attack ID is $v_{0} .2$ ) All node ID are put into set $V$. 3) Forge a node ID $v_{k} \notin V$. 4) select any node $v_{i} \in V$ and find a route $R_{0, i}$ from attacker $v_{0}$ to $v_{i}$. 5) Replace $v_{i}$
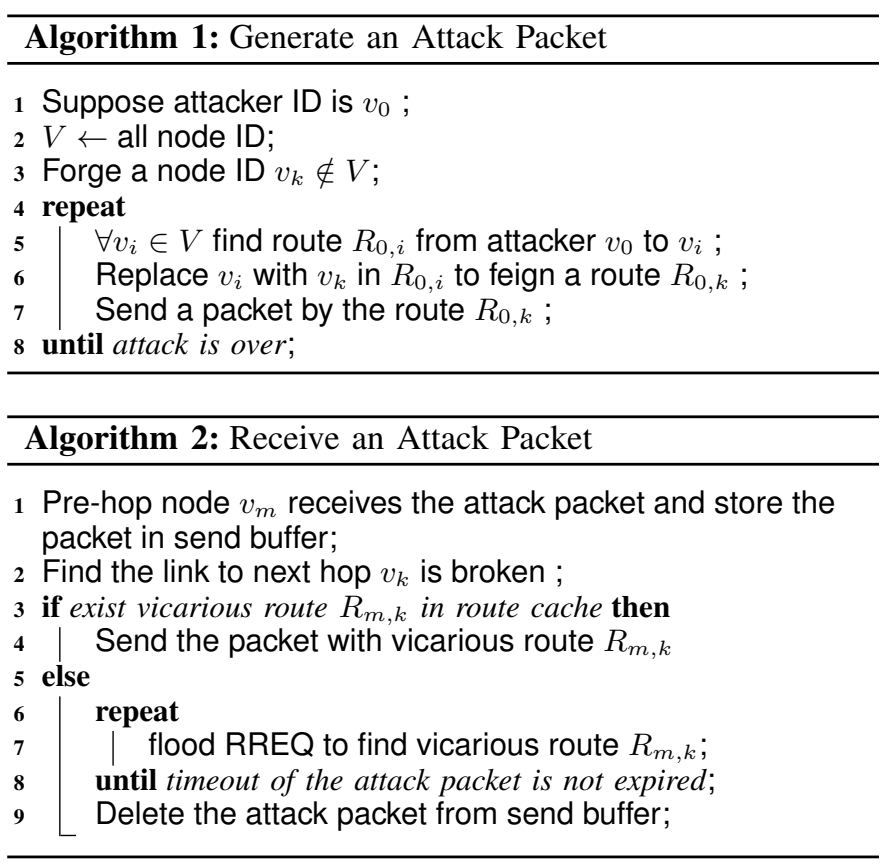

with $v_{k}$ in $R_{0, i}$ to feign a route $R_{0, k}$. 6) Send a packet by the route $R_{0, k}$. If attack is not over, go to step 4 .

Algorithm 2 shows the salvage process when a node receives an attack packet. Suppose when one node $v_{m}$ receives the attack packet, it attempts to forward it according to the specified route to the next hop node $v_{k}$. But $v_{m}$ finds it cannot reach $v_{k} . v_{m}$ starts to salvage the packet by searching in its cache for a placement route to $v_{k}$. If $v_{m}$ can find a route, it replaces the original route in the packet with the route from its route cache. Then $v_{m}$ sends the packet to the next node indicated by this route. If it cannot find route in its cache, $v_{m}$ starts to broadcasts a RREQ to find a new route to the destination. Because $v_{k}$ is not in the network, $v_{m}$ cannot receive a RREP from $v_{k} . v_{m}$ will repeatedly broadcast RREQs until the packet cache times out. If attacker $v_{0}$ sends many data packets that have specific error routes to $v_{m}, v_{m}$ will flood the network with many RREQs. As a result, $v_{m}$ starts the a flooding attack instead of the malicious attacker $v_{0}, v_{m}$ becomes a puppet of $v_{0}$. The attacker can also select other nodes as puppets and send attack packets which have specific error route to these puppets at the same time or in sequence.

In the most extreme example, the attacker first broadcasts RREQ packets to find routes from itself to all nodes in the network. The attacker changes every route into an error route just as in the above the example, and the attacker puts the error routes into packets and sends them out. The attacker carefully attacks so that all nodes in the network will start to salvage those packets which have the error routes. This will immediately result in the collapse of the network. Fig. 3 shows the effect of puppet attack on the network. Assume that Node $\mathrm{H}$ is an attack node. $\mathrm{H}$ sends four packets with error routes to its neighbors, and these packets result in neighbors $D, F, I, G$ salvaging the packets and broadcasting RREQ packets. Node $\mathrm{H}$ may send other packets with error routes resulting in 


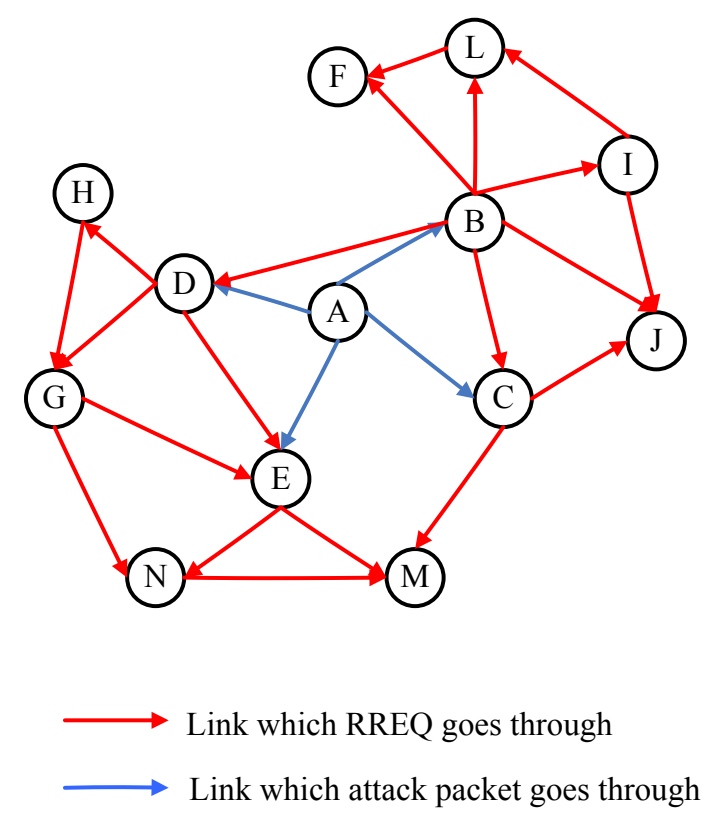

Fig. 3. The effect of puppet attack in a network

the other nodes $A, B, E, M, J, O, N, K$, and $C$ to start to salvage process. The flooded RREQ packets will exhaust the communication bandwidth and the nodes resource such as $\mathrm{CPU}$ and storage resource at the same time. For example, the storage of route tables in one node is limited. If RREQ packets are arriving to the node at a high rate, the route table in the node will be filled up so that the node cannot receive any new RREQ packet. As a result, normal nodes cannot build paths to send data. Fig. 3 shows that effect of puppet attack.

\section{Comparison Between Puppet Attack and Flooding Attack}

Based on the above description, puppet attack is similar to flooding attack. They all are DoS attacks and result in flooding RREQ packets throughout the network. However, puppet attack differs from flooding attack. Table 1 shows the difference between puppet attack and flooding attack.

TABLE I

CONTRAST BETWEEN PUPPET ATTACK AND FLOODING ATTACK

\begin{tabular}{lll}
\hline Name & puppet attack & flooding attack \\
\hline Attacker & $\begin{array}{l}\text { The normal nodes } \\
\text { which are selected as }\end{array}$ & The intruder itself \\
& puppet nodes & \\
\hline Attack method & $\begin{array}{l}\text { The intruder selects } \\
\text { some nodes as pup- }\end{array}$ & $\begin{array}{l}\text { The intruder floods } \\
\text { pets and sends at- } \\
\text { packets by itself } \\
\\
\text { tack packet to these }\end{array}$ \\
& $\begin{array}{l}\text { puppets. The puppet } \\
\text { nodes flood RREQ }\end{array}$ \\
& packets & \\
\hline Goal & Denial of service & Denial of service \\
\hline
\end{tabular}

The common goal of these two attacks is to achieve denial of service, but their attack methods are different. Therefore, puppet attack is a new attack model in mobile ad hoc networks.
Based on the above description, the puppet attack exhibits two features: secrecy and efficiency. The puppet attack is more difficult to detect. Acting as a normal node, the malicious node only sends attack packets that have specific routes when the malicious node starts to attack. The attack packets are the same as normal data packets except that they contain error routes. Other nodes cannot distinguish between correct and error routes in the attack packets since the source node (i.e. malicious node) creates the error route. Malicious nodes need not send many attack packets to control their puppets. The puppets broadcast excess RREQ packets to attack the network. Therefore, it is very difficult to detect the malicious node.

In the most extreme example, the malicious node first broadcasts a RREQ to find the routes from itself to all nodes in the network. The malicious node then changes every discovered route into an error route as in the above examples, places them into packets, and sends them. The malicious node will send these attack packets at the same time so that all nodes in the network will start the route salvage. The attack packets will result in all normal nodes inside the network acting as puppets and broadcasting RREQ packets. This can result in a collapse of the network. Therefore, the effect of puppet attack is more serious than that of flooding attack.

\section{The Approaches to Detect And Prevent Puppet ATTACK}

In this section, we describe a mechanism to efficiently detect and prevent the puppet attack. The method contains two steps: detection and prevention. In the first step, we design a distributed method to detect the puppet attack. In the second step we design a mechanism called link cut-off to isolate the attacker.

\section{A. Detecting Puppet Attack}

During route maintenance of DSR protocol, when a node sending a packet along a specified route to the destination discovers that the route has broken, it returns a route error (RRER) to the source of the packet, identifying the path from itself to the next-hop node as broken. The attacker sends many attack packets with specific routes to puppet nodes, which cannot get to their destinations for the specific routes in the packets. These puppet nodes then return RRERs to the attacker according to the route maintenance mechanism. Therefore, the attacker will receive many RRERs after it starts the puppet attack. The main idea of detection is that each node monitors the rate at which RRERs returned to the attacker.

Because wireless communication is broadcasting, nodes can hear packets broadcasted by its neighbor nodes. Node can keep track of the number of RRER packets returned to its neighbor nodes. We take Fig. 4 as an example. Node D is an attacker. Node A can receive the RRER packets returned to D from B and C. Similarly, Node B can receive the RRER packets returned to D from A.

Each node sets up a counter to record the RRER rate for packets to its neighbors. This counter should be a dynamic 


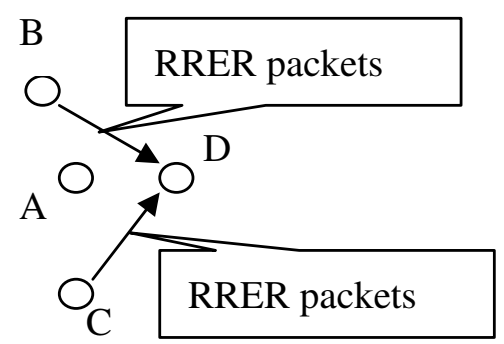

Fig. 4. The node hears RRER packets returned to its neighbor node

value depended on the rate of route broken in a network. It not only shows current RRER rate, but also should reflect historic records. Therefore, we define the value as $P(i, t)$, which is a cumulative average value of RRERs returned to neighbor node $i$ at time $t$. It can be calculated as follows:

$$
\begin{array}{r}
P(i, t)=\gamma Q(i, t)+(1-\gamma) P(i, t-1) \\
i \in Z ; \quad 0<\gamma<1
\end{array}
$$

where $i$ is the node ID. $Z$ is the set of neighbors. $t$ is a continuous time like the fifth second. $\gamma$ is a weight factor used to trade off between the current measurement and the previous calculation. $Q(i, t)$ is the number of RRERs to neighbor node $i$ during time interval $(t-1, t]$. We get the cumulative average value $P(i, t)$ of each neighbor nodes from formula ( 1$)$. Then in order to know the RRER rate in the whole networks, we calculate the average value of RRER packets of all neighbors at time $t$. We define the value as $D(t)$, which can be calculated as follows.

$$
D(t)=\sum_{i \in Z} \frac{P(i, t)}{m}
$$

where $D(t)$ is the total average RRERs rate to all neighbors at time $t . m$ is the total number of nodes in set $Z$.

We introduce a threshold $H(t)$ as follows.

$$
H(t)=\max (\alpha D(t), \beta)
$$

where $\alpha$ is a factor to adjust the threshold to detect a puppet attack. It affects the detection rate and false alarm rate. We set $\alpha=3$ in our simulations by experience. $\beta$ is a factor to avoid the extremely small threshold. For example, if no $\beta$, an exception occurs under most nodes received a little or no RRER. i.e. $D(t) \approx 0$ and $H(t) \approx 0$. In this setting, if any a node receives an RRER and the other neighbor nodes do not receive any RRERs, $P(i, t)=1$ and $D(t)=\frac{1}{m}$ from formula (2). If $\alpha<m$, we then get $Q(i, t)>H(t)$ and node $i$ will be regarded as the attacker. Therefore, we introduce a second threshold $\beta$ to avoid the above problem. Simulations show that the number of RRERs return one node changes from 0 to 7 without attacker. And therefore we set up $\beta=10$ in our simulations. If $Q(i, t)$ of one node exceeds the threshold in formula ( 3 ), the ID of its node will be put into the blacklist of his neighbor nodes.
Every node respectively calculates the threshold in formula ( 3) by its forwarded RRER records. Every node respectively makes a judgement by its threshold. Nodes do not need to exchange any information with each other. It reduces network traffic and meanwhile it also prevents malicious nodes to disseminate false information.

\section{B. Preventing Puppet Attack}

The features of WSNs are multi-hop and wireless communication. A node receives and sends packets through its neighbor nodes. A node does not have connection with the other nodes in the network, if its all neighbors cut off their links to it. The attacker has actually been isolated from the network even if it is still physically located in the network. Fig.5 shows how to isolate the attacker in WSNs. Node $H$ communicates with other nodes through node $I, G, F$ and $D$. If neighbor node $I, G, F$ and $D$ refuse to receive packets from node $H$, node $H$ is isolated and cannot send any packet to the other nodes.

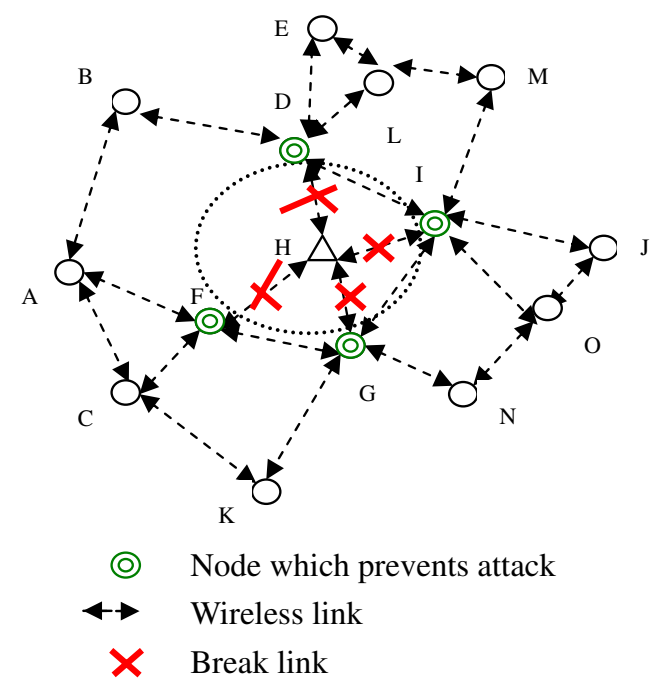

Fig. 5. The Neighbor nodes isolate attacker

Every node detects the puppet attack by receiving or overhearing RRER packets. These nodes put some node ID into their blacklist if they find it is the puppet attacker. These nodes firstly look source ID of the packet in their blacklist when these nodes receive a packet. These nodes directly discard the packet if the source ID is in their blacklist. If source ID of the packet is not in their blacklist, these nodes forward the packet by the normal process.

These nodes do not broadcast their blacklist. And they do not receive the other node's blacklist. They detect the attacker by themselves. We take Fig. 5 for example. Assume node $H$ is an attacker and sends attack packets to node $K$ through $G$. $K$ will return many RRERs to $H$ through node $G$, for $K$ cannot forward these attack packets. Neighbor $G$ finds that node $H$ receives too many RRERs and RRER's frequency exceeds the threshold $H$ by formula ( 3 ). $G$ will put $H$ into 
its blacklist and drop all packets from $H$. $H$ finds that it cannot send attack packets to $K$ through $G$. $H$ changes another route $H-F-C-K$ to send attack packets to $K . K$ returns many RRERs through route $K-C-F-H$ to $H$ soon. $F$ will find the number of RRERs to $H$ exceeds the the threshold. $F$ will put $H$ into the blacklist and cut off the link from $F$ to $H$. Similarly, if $H$ continues to send attack packets by other routes, $I$ and $D$ will find $H$ is attacker and do not receive any packets from $H$. As a result, the puppet attack from node $H$ is prevented by its neighbors in Fig.5.

Every node independently detects and prevents the puppet attack. It has some advantages. The first point is to reduce the network traffic, for every node does not exchange information like blacklist packet. The second point is to avoid fraud information. For example, malicious node broadcasts false blacklists to isolate a normal node. But the method has some disadvantages. It takes a longer time to isolate the attacker. Every neighbor node to an attacker has to detect the puppet attacker by itself.

\section{Evaluation}

In this section, we introduce the experiment setup, describe the simulation results, and discuss the reason behind the simulation results.

\section{A. Experimental setup}

To study the severity of the puppet attack, we have implemented puppet attack in ns-2 (an Network Simulator). In our simulation, 50 wireless nodes are randomly deployed in a 1000 meter by 1000 meter flat space. The total duration of the simulations is 400 seconds. Each data point on a graph represents the average value of 30 runs.

We use packet delivery rate to indicate the performance of puppet attack. Packet delivery rate is the ratio between the number of packets originated by the source nodes and the number of packets received by the final destination nodes. Packet delivery rate represents the maximum throughout that the network can achieve.

\section{B. Simulation results of puppet attack}

The system performance has been observed in various scenarios including attacking frequency and attacking nodes. The first scenario is performance under different numbers of puppets with one attacker. One attacker sends 50 packets with a forging route. The number of puppet nodes is $1,5,10,15,25$, and 50. The performance of packet delivery rate is in Fig.6. $\mathrm{N}$ is the number of puppets. An attacker selects from 1 to 50 nodes as puppets. The attacker starts to send data packet at $50 \mathrm{~s}$. When the attacker selects one node as a puppet, the packet delivery rate will go down to 30 percent. With the increase of the number of puppets, the packet delivery rate will continue to decline. When the attacker selects ten nodes as the puppet, the packet delivery rate will go down to 18 percent. This is the most efficient number of puppet nodes. Then with the increase of the number of puppets, the packet delivery rate will not decrease and begin to increase. When the number of puppets is 50 , the packet delivery rate goes up to 35 percent and the performance is better than that under one puppet. The main reason is that many attacking packets may not arrive at their puppet nodes because there are too many puppets under puppet attack.

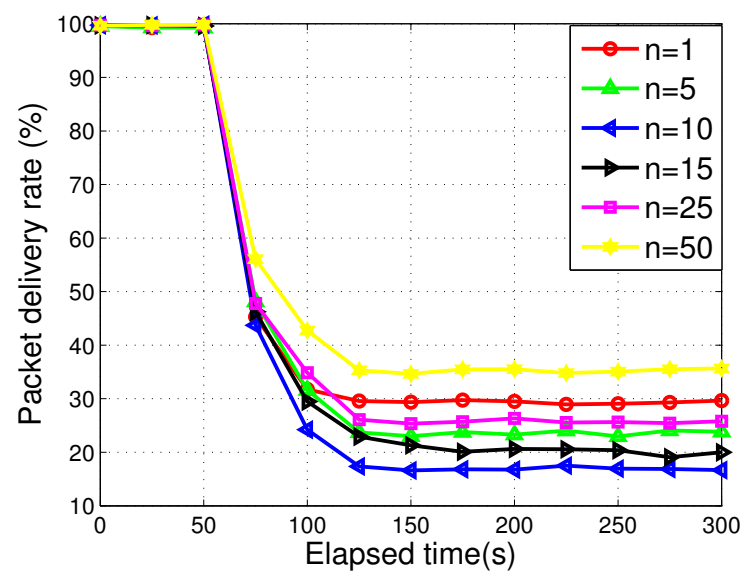

Fig. 6. The packet delivery rate under different number of puppets

The second scenario is performance under different numbers of attackers with the same frequency. The number of attackers is from 1 to 5 . Every attacker selects one node as the puppet and sends 10 attacking packets every second. Five attacker nodes are static and their locations are $(500,500),(250,250)$, (750,750), $(250,750),(750,750)$ in 1000 meter by 1000 meter flat space.

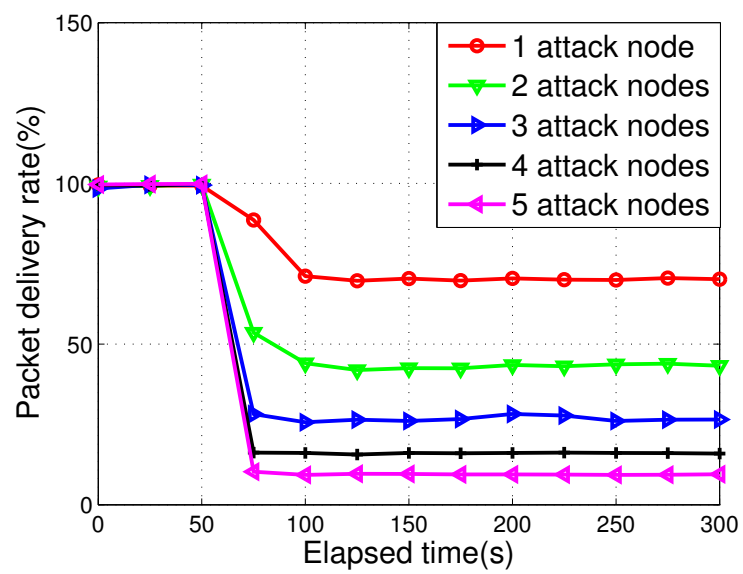

Fig. 7. The packet delivery rate under different number of attack nodes

Fig.7 shows that the packet delivery rate when the number of attack nodes varies from 1 to 5 . The attacker starts to send data packets at $50 \mathrm{~s}$. When one attacker selects one node as a puppet and sends 10 attacking packets every second, the packet delivery rate will go down to 70 percent. When three attackers select three nodes as puppets and send 30 attacking packets every second, the packet delivery rate will go down 
to 27 percent.

Compare to the first scenario, when one attacker selects one node as a puppet and sends 50 attacking packets every second, the packet delivery rate will go down to 30 percent in Fig.6. However, we observe the difference is that one attacker sends 50 attacking packets every second in the first scenario and three attackers send 30 attacking packets every second in the second scenario. Therefore, multi-attacker will result in the same effect of the performance of a network with low frequency of attacking packets. When five attackers send 50 attacking packets every second, the packet delivery rate will go down to 10 percent. This is the most efficient attack result in the first scenario is that the packet delivery rate will go down to 18 percent with ten nodes as puppets. Furthermore, the effect of multi-attackers is more serious than that of one attacker. It is because the attacking packets which multi-attackers send may arrive the puppets. Conversely, the attacking packets that sent by one attacker may be blocked and dropped due to mass RREQ packets.

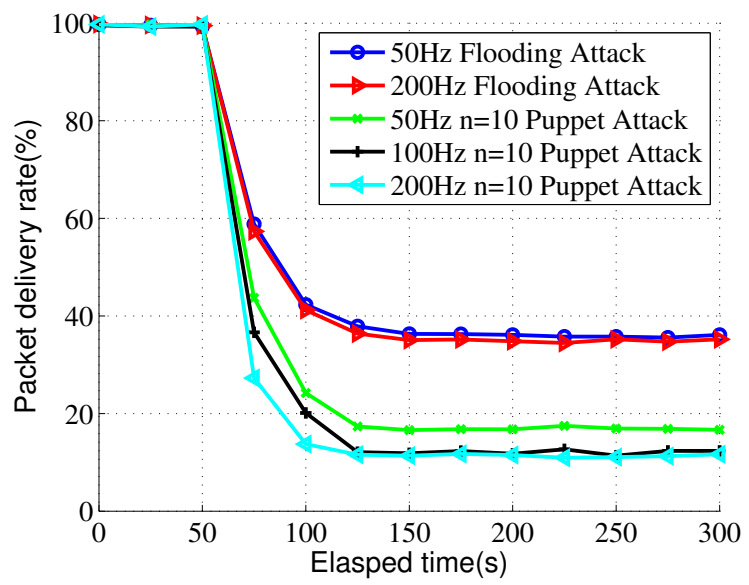

Fig. 8. The packet delivery rate at one attacker with different attack frequency between puppet attack and Flooding Attack

We compare puppet attack with Flooding Attack in the following scenarios under various parameters. The third scenario is performance between puppet attack and Flooding Attack at one attacker with different attack frequency. Fig.8 shows that one attacker broadcast 50 RREQ packets and 200 RREQ packets every second in Flooding Attack. One attacker selects ten nodes as puppets and sends 50 attacking packets, 100 attacking packets and 200 attacking packets. The effect of puppet attack is two times even three times than that of Flooding Attack in the same attack frequency. The simulation shows that the packet delivery rate will go down to 17 percent in puppet attack and the packet delivery rate will go down to 36 percent in Flooding Attack when the frequency of attacking packets is 50 packets every second. The packet delivery rate will go down to 11 percent in puppet attack and the packet delivery rate will go down to 34 percent in Flooding Attack when the frequency of attacking packets is 200 packets every second. The effect of puppet attack is two times even three times than that of Flooding Attack in the same attack frequency. The attacker who broadcasts RREQ packets in high frequency will result in congestion in neighbor nodes of the attacker. A lot of RREQ packets will be dropped by neighbor nodes for the congestion in Flooding Attack. However, an attacker selects ten nodes as puppets in puppet attack. The ten puppets broadcast RREQ packets instead of the attacker just as ten attackers. The effect of puppet attack is more serious than that of Flooding Attack.

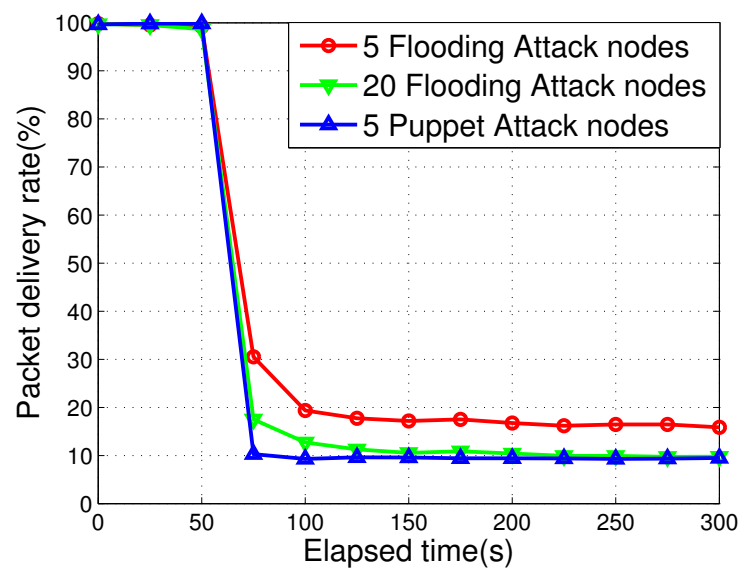

Fig. 9. The packet delivery rate at various attackers with the same attack frequency between puppet attack and Flooding Attack

The fourth scenario is performance between puppet attack and Flooding Attack at various attackers with the same attack frequency. Fig.9 shows that the attack frequency is 10 attack packets per second. Namely, one attacker will send 10 RREQ packets every second with regard to Flooding Attack. And one attacker will send 10 packets with error route every second with regard to puppet attack. The various parameters are the number of attack nodes. Fig.9 shows that the packet delivery rate will go down to 17 percent when 5 attack nodes flood RREQ packets at 10 attack packets per second. When the number of attack nodes gets to 20 nodes, the packet delivery rate will go down to 10 percent. By contrast, there are 5 puppet attack nodes will result in the same attack efficiency under the same attack frequency. The simulation shows that the packet delivery rate will go down to 10 percent when 5 puppet attack nodes start to puppet attack. The main reason is that puppet attack nodes may select the other nodes in the network as puppets. Although there are only 5 attackers in the network, they will let tens of puppets to flood RREQ packets. Therefore, the effect of puppet attack is four times than that of Flooding Attack in the same attack frequency.

Based on the above simulations and analysis, puppet attack will result in more performance degradation than Flooding Attack, because attackers can select more nodes as puppets to start to attack network. The effect of puppet attack will be three or four times than that of Flooding Attack under the same parameters. Similarly, because attackers can select normal nodes as puppets to attack the network instead of 
themselves, the puppet attack is more difficult to detect than Flooding Attack.

\section{Simulations of Attack Detection}

In this section, we analyze the simulations for our proposed method presented in above sections to detect the puppet attack. The neighbor nodes monitor the RRER rate returned to the attacker. If the RRER rate exceeds the formula( 3 ) in Section III, the attacker will be put into the blacklist.

We set up $\gamma=0.7$ and $\beta=10$ by experience. $\gamma=0.7$ denotes that current number is more important than historic records. $\beta=10$ means that the node is not regarded as an attacker if it receives less than 10 RRERs within one second. $\alpha$ represents that the threshold of detection, which is the key parameter for detection. We setup $\alpha$ value from 2 to 6 . For example, $\alpha=3$ represents the threshold of detection is triple of the average RRER rate.

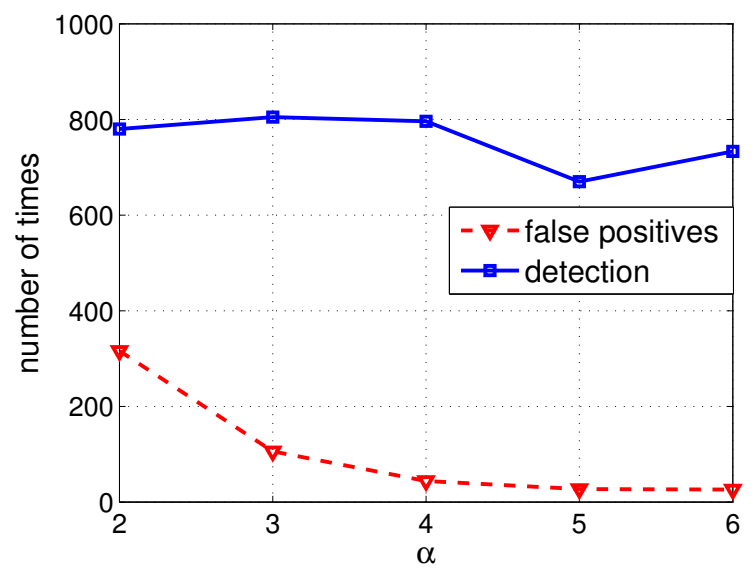

Fig. 10. The number of detection and false positives for puppet attack

Fig. 10 shows that the number of detection and false positives for puppet attack. The number of detection is the time which our method detects the puppet. One intruder starts puppet attack one time. The number of detection is one if one node detects the attack. If there are three neighbor nodes to the attacker and they all detect puppet attack, the number of detection is three. If one intruder starts puppet attack three times and three nodes all detect these attack three times, the number of detection is $3 * 3=9$. If one node reports a puppet attack and there is no puppet attack now, the false positives is one.

When $\alpha$ value varies from 2 to 6 , the number of detection change a little and vary between 700 and 800 . But the number of false positives falls very quickly. When $\alpha=2$ represents the threshold of detection is two times of the average RRER rate, the number of false positives is 316. When $\alpha=3$, the number of false positives is 106 and is one-third of 316 . When $\alpha=4$, the number of false positives is 44 and is one-half of last false positives. When $\alpha=5$, the number of false positives is 27 . It decreases about one half of 44 .
These results show that the number of false positives will decrease with increase of the detection threshold $\alpha$. If $\alpha$ is 2 or 3 , the detection method will report puppet attack when there are a few RRER packets. These RRER packets may be returned by route broken and they are not the result of puppet attack. When $\alpha$ is 5 or more, the detection method will neglect some RRER packets which are not produced by puppet attack. With increase of $\alpha$, the detection method also fails to detect some puppet attacks. For example, when $\alpha=5$ the number of detection is 670 and it is less than the number 796 with $\alpha=4$. But the decrease of the number of detection is little.

\section{Simulations of Attack Prevention}

In this section, we do some simulations to show prevention effect under attack prevention and no prevention. There is no puppet attack from 0 to $50 \mathrm{~s}$. The puppet attack starts from 50 s to 300 s. In this simulation, there is one attacker which sends 50 attack packets every second in the networks. There are functions of detecting puppet attack in each node.

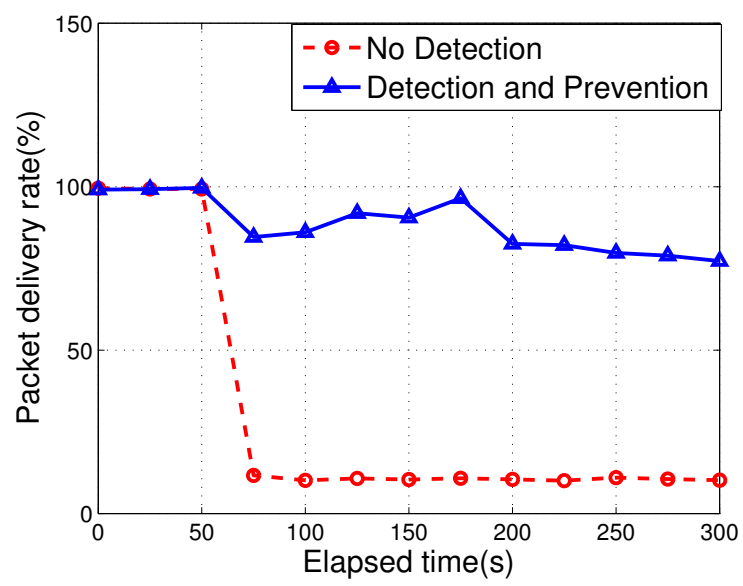

Fig. 11. The packet delivery rate under no prevention and attack prevention

Fig. 11 shows that the packet delivery rate under no prevention and attack prevention. If there is no detection and prevention, the packet delivery rate falls to about $10 \%$ for the puppet attack results in network congestion. If there is detection and prevention, the packet delivery falls a little and stays between $77 \%$ and $91 \%$. The main reason is that the attacker still affects some nodes even if it is isolated by neighbors. We take Fig.5 for example. $H$ is isolated by its neighbors, but the continual attack from $H$ can still affect communication in the area of $D, I, F, G$. For example, if $E$ plans to send a packet to $G, E$ have to select a longer route $E-D-B-A-F-G$ instead of route $E-D-H-G$, for the shorter route is blocked by attacker $H$. Even so, network performance has improved significantly and packet delivery rate increases from $10 \%$ to about $85 \%$. It shows that the mechanism to detect and prevent the puppet attack is efficient. 


\section{RELATED WORK}

In our previous work [7], we only present a DoS attack in Advanced Metering Infrastructure Network, but there is not any detection and prevent method. In this paper, we add one section on the approaches to detect and prevent puppet attack to discuss how to detect and prevent the puppet attack in this paper. We present a a distributed detection way to monitor the number of RRER packets to the attacker. We design an adaptive prevention mechanism which the neighbor nodes cut off the routes to the attacker as they detect the puppet attack. And then we do some simulations to show these methods efficiently.

We also discuss potential security attacks and possible counter-attack measures in smart grid, and present a new intrusion detection and response scheme to meet the special requirements of wireless network in smart grid [8], [9], [10], [11]. In paper [8], a wireless mesh network-based wireless security architecture was proposed for smart grid. In paper [9], an energy efficient intrusion prevention mechanism is designed in wireless mesh network. In paper [10], we develop a novel secure energy routing mechanism for securely and optimally sharing renewable energy in smart microgrids, which detects most internal attacks by using message redundancy. In paper [11], we develop an intrusion prevention mechanism in mobile ad hoc networks called mobile firewall. It can isolate an intruder with less overhead, and it can track the intruder to continually prevent the attack. Papers [8], [9], [10], [11] discuss some security mechanisms, and this paper discusses a DoS attack and defense in Advanced Metering Infrastructure Network.

A key management framework is presented for AMI networks in smart grid, which is a unified key management mechanism that can generate ciphering keys for multiple protocols of multiple communication layers from a single peer entity authentication procedure [12]. J. Kamto designs a framework for key distribution and management for both aggregation and accountability in a neighborhood area network employed by a utility company in its power distribution system [13]. An exposure metric has been proposed to identify the set of security mechanisms required to protect the various information objects utilized within an AMI network [14]. An adaptive tree inspection scheme that employs the heuristic information is discussed for malicious meter inspection problem in neighborhood area smart grids [15]. C. Fan presents a privacy enhanced data aggregation scheme against internal attackers for smart grids and provided formal security [16].

An automated AMI configuration verification, diagnosis and repair technique that is presented, which creates a logic-based formal model of AMI behavior based on AMI device configurations and verifies the compliance of this model with system invariants and security requirements using constraint satisfaction checking [17]. Authors have investigated applicable communication mechanisms that could be adopted on smart grid distribution networks. They further elaborated on their practical feasibility in terms of their technical implementation, possible obstacles, and core security issues, and attacks on smart grid [18].

\section{CONCLUSION}

Smart grid has received significant attentions in recent advances. AMI network in smart grid delivers electricity from providers to users, and it uses two-way digital communications to control appliances at users homes. In this paper, we identified and described the puppet attack, a new and severe denial of service attack in AMI network. We analyze the model of the puppet attack and compare it with flooding attack. The puppet attack is more difficult to detect than flooding attack. We also conducted simulations to investigate the effect of the puppet attack. The simulation results indicate that the puppet attack result in a serious decline in AMI network performance. After analyzed the puppet attack, we present a detection and prevention method to detect the attack. The simulations show the method can detect and prevent the puppet attack efficiently.

\section{ACKNOWLEDGEMENT}

This research was supported in part by National Key Basic Research Program of China(No.2013CB329603), the National Natural Science Foundation of China (No.61431008, No.61271220, No.61170164), the Natural Science Foundation of Shanghai (No.15ZR1423600), NSF grant CNS-1217791, Information content security management innovation base (TS0010303001), State Engineering Laboratory for Information Content Analysis Technology (GT036001)

\section{REFERENCES}

[1] S. Grillo, V. Musolino, L. Piegari, E. Tironi, C. Tornelli, DC Islands in AC Smart Grids, IEEE Transactions on Power Electronics, 2014, Vol.29, No.1, pp.89-98.

[2] T.A. Short, Advanced Metering for Phase Identification, Transformer Identification, and Secondary Modeling, IEEE Transactions on Smart Grid, March 2013, VOL.4, NO.2, pp.651-658

[3] P. Kulkarni, S. Gormus, Z. Fan, B. Motz, A Mesh-Radio-Based Solution for Smart Metering Networks, IEEE Communication Magazine, 2012, Vol.38, No.7, pp.86-95.

[4] H. Gharavi, C. Xu, Distributed Application of the Traffic Scheduling Technique for Smart Grid Advanced Metering Applications Using MultiGate Mesh Networks, in IEEE GLOBECOM, 2011

[5] D. Grochocki, J. H. Huh, R. Berthier, R. Bobba, W. H. Sanders, A. A. Cardenas, J. G. JetchevaRF Mesh Systems for Smart Metering: System Architecture and Performance, 2012 IEEE Third International Conference on Smart Grid Communications (SmartGridComm), 2012, pp.395 - 400

[6] D. Grochocki, J. H. Huh, R. Berthier, R. Bobba, W. H. Sanders, A. A. Cardenas, J. G. Jetcheva,AMI Threats, Intrusion Detection Requirements and Deployment Recommendations, in IEEE SmartGridComm,2012

[7] P. Yi, T. Zhu, Q. Zhang, Y. Wu, J. Li, A Denial of Service Attack in Advanced Metering Infrastructure Network, 2014 IEEE International Conference on Communications (ICC2014), Sydney, Australia,10-14 June 2014

[8] X. Wang, P. Yi, Security Framework for Wireless Communications in Smart Distribution Grid, IEEE Transactions on Smart Grid, 2011, Vol.2, No.4, pp.809 - 818

[9] P. Yi, T. Zhu, Q. Zhang, Y. Wu, J.H Li, Green Firewall: an EnergyEfficient Intrusion Prevention Mechanism in Wireless Sensor Network, In IEEE GLOBECOM, 2012

[10] T. Zhu, S. Xiao, P. Yi, D. Towsley, W. Gong, A Secure Energy Routing Protocol for Sharing Renewable Energy in Smart Microgrid, In SmartGridComm, 2011

[11] P. Yi, T. Zhu, J.Q Ma, Y. Wu, An Intrusion Prevention Mechanism in Mobile Ad Hoc Networks, Ad Hoc \& Sensor Wireless Networks, Vol.17, No.3-4, 2013, pp.269-292 
[12] S.Das, Y. Ohba, M. Kanda, D. Famolari, SK. Das, A Key Management Framework for AMI Networks in Smart Grid, IEEE Communication Magazine, 2012, Vol.38, No.4, pp.30-37.

[13] J. Kamto, L. Qian, J. Fuller, J. Attia Key Distribution and Management for Power Aggregation and Accountability in Advance Metering Infrastructure, in SmartGridComm, 2012

[14] A.Hahn,M. Govindarasu, Cyber Attack Exposure Evaluation Framework for the Smart Grid, IEEE Transactions on Smart Grid, 2011, Vol.2, No.4, pp. $835-843$

[15] A. Wood, J. A. Stankovic, Exploring Malicious Meter Inspection in Neighborhood Area Smart Grids, IEEE Transactions on Smart Grid, March 2013, VOL.4, NO.1, pp.214-226

[16] C. Fan, S. Huang, Y. Lai, Privacy Enhanced Data Aggregation Scheme Against Internal Attackers in Smart Grid, IEEE Transactions on Industrial Informatics, 2013, Vol.99

[17] M. A. Rahman, E. Al-Shaer, P. Bera, A Noninvasive Threat Analyzer for Advanced Metering Infrastructure in Smart Grid, IEEE Transactions on Smart Grid,, March 2013, VOL.4, NO.1, pp.273-287

[18] E. Bou-Harb, C. Fachkha, M. Pourzandi, M. Debbabi, C. Assi, Communication Security for Smart Grid Distribution Networks, IEEE COMMUNICATIONS MAGAZINE, 2013, Vol.51, No.1, pp.42-49. 